\title{
New Viewpoints about Pseudo Measurements Method in Equality-Constrained State Estimation
}

\author{
Bingjie Zhu, Yingting Luo, and Yunmin Zhu \\ College of Mathematics, Sichuan University, Chengdu, Sichuan 610064, China \\ Correspondence should be addressed to Yingting Luo; lyt83@163.com
}

Received 4 October 2014; Revised 20 February 2015; Accepted 25 February 2015

Academic Editor: Elmetwally Elabbasy

Copyright ( 2015 Bingjie Zhu et al. This is an open access article distributed under the Creative Commons Attribution License, which permits unrestricted use, distribution, and reproduction in any medium, provided the original work is properly cited.

\begin{abstract}
We discuss the pseudo measurement method which is one of the main approaches to equality-constrained state estimation for a dynamic system. We demonstrate by the fundamental theory of Kalman filtering that reviewing the equality constraint as a pseudo measurement seems questionable. The main reason is that the additional pseudo measurement is actually a constant here which cannot help to estimate the state. More specifically, when the states in an unconstrained dynamic system model have already satisfied the equality constraint, the extra constraint is obviously not necessary. When the true equality-constrained states do not satisfy the unconstrained dynamic process equation, the effect of pseudo measurement is projecting the estimate which is not optimal onto the constraint set. However, since the performance of a projected estimate is also certainly influenced by its original estimate, we show through a numerical example that the pseudo measurement method is not always a good choice, especially when the process equation mismatch is large.
\end{abstract}

\section{Introduction}

In practice, filtering problems often have certain inherent and known constraints in the physical dynamic systems [1], for example, target tracking [2,3], robotics [4], multisensor data fusion $[5,6]$, vision-based systems [7], econometric modeling [8], biomedical systems [9] and others [10, 11]. For equality-constrained state estimation, numerous approaches have been developed, for example, the model reduction method $[5,6,12]$, the pseudo measurement method [13-18], the estimate projection method $[19,20]$, the system projection method [21], the gain projection method [22], and some other methods $[11,23]$.

Among these methods, the pseudo measurement method has been deeply investigated. This method is to augment the measurement space of the filter with the equality constraints (i.e., as perfect noise-free measurements) at each iteration. It is clearly simple and easy to be implemented. However, since the pseudo measurements are error free, the augmented measurement noise has a singular covariance. Numerical problems may arise when the Kalman filtering is applied. Moreover, the increase in the dimension of the augmented measurement will increase the computational burden of the state estimator [20]. In order to deal with this problem, Duan and Li proposed two sequential forms of the pseudo measurement method in [18]. By the sequential forms, they computed the equivalence of Kalman filtering with and without pseudo measurement and proved that, under certain conditions, the use of the pseudo measurement for filtering is redundant. There are also many discussions about the equivalence between the pseudo measurement method and the others (see [17, 24-26]). Gupta [24] proved that, under certain conditions, the pseudo measurement method and the estimate projection method result in mathematically equivalent constrained estimate structures. Moreover, this equivalent estimate has a smaller error covariance than that of the unconstrained state estimate [20].

In this paper, we discuss the reasonability of the pseudo measurement method. By the fundamental theory of Kalman filtering, we demonstrate that reviewing the equality constraint as a pseudo measurement seems questionable. The main reason is that the additional pseudo measurement is actually a constant here which cannot help to estimate the state. More specifically, when the states in an unconstrained dynamic system model have already satisfied the equality constraint, the extra constraint is obviously not necessary. 
When the true equality-constrained states do not satisfy the unconstrained dynamic process equation, but they are actually observed, that is, the true state only satisfies the measurement equation, the optimality of Kalman filtering is of course lost. The effect of pseudo measurement in this case, as said by Gupta [24], is to project the estimate which is not optimal onto the constraint set. Therefore, when such an estimate is outside the constraint set, the projected estimate will of course be better than the Kalman filtering without the pseudo measurement. Our analysis seems more straightforward and essential. Moreover, since the performance of a projected estimate is also certainly influenced by its original estimate, we show through a numerical example that the pseudo measurement method is not always a good choice especially when the process equation mismatch is large.

This paper is organized as follows. In Section 2, problem formulation and a brief summary of the pseudo measurement method are presented. In Section 3, we demonstrate that the pseudo measurement method is redundant simply by using the fundamental theory of Kalman filtering rather than computing the equivalence of Kalman filtering with and without pseudo measurement. We also discuss the pseudo measurement method in the mismatch process equation. Numerical examples are provided in Section 4. Finally, we present our conclusions in Section 5.

\section{Problem Formulation}

Consider the following dynamic system:

$$
\begin{gathered}
x_{k+1}=F_{k} x_{k}+v_{k}, \\
y_{k}=H_{k} x_{k}+\omega_{k},
\end{gathered}
$$

where $x_{k} \in \mathscr{R}^{m}$ is the system state, $y_{k} \in \mathscr{R}^{n}$ is the measurement vector, and the process noise $\nu_{k} \in \mathscr{R}^{m}$ and measurement noise $\omega_{k} \in \mathscr{R}^{n}$ are zero-mean white noise sequences with the following covariances

$$
\begin{array}{r}
E\left(v_{k} v_{j}^{T}\right)=R_{v_{k}} \delta_{k-j}, \quad E\left(\omega_{k} \omega_{j}^{T}\right)=R_{\omega_{k}} \delta_{k-j}, \\
E\left(v_{k} \omega_{j}^{T}\right)=0, \quad E\left(v_{k} x_{0}^{T}\right)=0, \quad E\left(\omega_{k} x_{0}^{T}\right)=0, \\
\forall k, j,
\end{array}
$$

where $\delta_{k-j}$ is the Kronecker delta function, that is $\delta_{k-j}=1$ if $k=j$ and $\delta_{k-j}=0$ if $k \neq j$. Matrices $F_{k}$ and $H_{k}$ are of appropriate dimensions. It is known that the state vector $x_{k}$ in (1) is subject to the following linear equality constraint:

$$
D_{k} x_{k}=d_{k}
$$

where $D_{k}$ is a known constant matrix and $d_{k}$ is a known constant vector.

To deal with this problem, the pseudo measurement method first augments the measurement space of the filtering with the equality constraints (i.e., as perfect noise-free measurements) at each iteration and rewrites the measurement equation (2) as partitioned form accordingly:

$$
\left(\begin{array}{l}
y_{k} \\
d_{k}
\end{array}\right)=\left(\begin{array}{l}
H_{k} \\
D_{k}
\end{array}\right) x_{k}+\left(\begin{array}{c}
\omega_{k} \\
0
\end{array}\right)
$$

and the state equation (1) is not changed. Then classic Kalman filtering can be used to estimate the state vector in the remodeled dynamic system (1) and (5). The details are omitted here.

Remark 1. We must notice that the modification from (2) and (4) to (5) is not equivalent. The state vector $x_{k}$ is random and its value depends on the sample of the process noise $v_{k}$. However, the equality constraint equation (4) of the state exists objectively. For example, a car moves on a road. It means that every possible sample of the state $x_{k}$ should satisfy the equality constraint. On the other hand, if we take the equality constraints as a pseudo measurement (5), it only requires the current measured sample of state $x_{k}$ to satisfy the equality constraint and does not restrict all possible values of $x_{k}$ in the constraint set. Therefore, the equality constraint has been changed significantly in this method.

In fact, the true state vector $x_{k}$ must satisfy the equality constraint equation (4). However, in practice, we often give the dynamic system equation (1) at first. Then, if we get extra information about the state vector, we give the additional constraint (4). In this situation, usually, $x_{k}$ in (1) cannot be guaranteed to satisfy the equality constraint equation (4). We call the process equation in this situation mismatch. In the following, we will discuss the pseudo measurement method in the cases of both an exactly matched process equation and a mismatched process equation.

\section{Analysis of Pseudo Measurement Method}

In this section, we will discuss the pseudo measurement method for more details. From Section 2, we can see that the essence of the pseudo measurement method is augmented measurement Kalman filtering. Kalman filtering is the best known recursive least mean square error (LMSE) algorithm to optimally estimate the unknown state of a dynamic system. For later use with analyzing the pseudo measurement method, we first present the famous LMSE estimate theorem (see [27]).

Lemma 2. Let the random variable

$$
\left(\begin{array}{c}
x^{T} \\
y^{T}
\end{array}\right)
$$

have mean and covariance

$$
\left(\begin{array}{l}
m_{x} \\
m_{y}
\end{array}\right), \quad\left(\begin{array}{ll}
\Sigma_{x x} & \Sigma_{x y} \\
\Sigma_{y x} & \Sigma_{y y}
\end{array}\right) .
$$

Then, the LMSE estimate of $x$ using $y$ is given by

$$
\widehat{x}=m_{x}+\Sigma_{x y} \Sigma_{y y}^{\dagger}\left(y-m_{y}\right)
$$

where $\dagger$ denotes the pseudoinverse.

We denote the following:

$\widehat{x}_{k \mid k}$ is the estimation of the dynamic system equations (1)-(2) by Kalman filtering; 
$\tilde{x}_{k \mid k}$ is the estimation of the augmented dynamic system equations (1), (5) by Kalman filtering, that is, the pseudo measurement method.

We have the following theorem for the pseudo measurement method with exactly matched process equation.

Theorem 3. If the two estimators are initialized by the same $x_{0}$ and error covariance matrix $P_{0}$, the use of the pseudo measurement for exactly matched process equation is redundant; that is,

$$
\widehat{x}_{k \mid k}=\tilde{x}_{k \mid k}
$$

Proof. When the process equation is exactly matched, the pseudo measurement method is just a recursive LMSE estimation using all observations $\left(y_{1}, \ldots, y_{k}, d_{k}\right)$ to estimate $x_{k}$. Since $d_{k}$ is a constant vector, $\operatorname{Cov}\left(x_{k}, d_{k}\right)=0$. According to Lemma 2 , it is no use to estimate $x_{k}$ by a constant vector $d_{k}$. Thus, it is easy to see that the pseudo measurement method, which adds a constant vector $d_{k}$ to estimate the state vector $x_{k}$, is redundant for the LMSE estimation; that is, $\widehat{x}_{k \mid k}=$ $\tilde{x}_{k \mid k}$.

Gupta [24] proved that under certain conditions, the pseudo measurement method and the estimate projection method result in mathematically equivalent constrained estimate structures. Moreover, this equivalent estimate has a smaller error covariance than that of the unconstrained state estimate [20]. This is to say the estimation $\tilde{x}_{k \mid k}$ given by the pseudo measurement method should satisfy the equality constraint equation (4) and its result is better than $\widehat{x}_{k \mid k}$; that is,

$$
D_{k} \tilde{x}_{k \mid k}=d_{k}, \quad \operatorname{cov}\left(x_{k}-\tilde{x}_{k \mid k}\right) \leq \operatorname{cov}\left(x_{k}-\widehat{x}_{k \mid k}\right) .
$$

By Gupta's conclusion, the estimate of pseudo measurement method, $\tilde{x}_{k \mid k}$ is the projection of $\widehat{x}_{k \mid k}$ onto the constraint set. However, we have $\widehat{x}_{k \mid k}=\tilde{x}_{k \mid k}$ in Theorem 3. In fact, Theorem 3 does not contradict Gupta's conclusion, since, in the following corollary, we show that $\widehat{x}_{k \mid k}$, without the pseudo measurement in Theorem 3 , has already satisfied the constraint.

Corollary 4. If the state vector in (1) has already satisfied the equality constraint equation (4), the estimate by Kalman filtering without the pseudo measurement must satisfy the constraint; that is, $D_{k} \widehat{x}_{k \mid k}=d_{k}$.

Proof. As we know, the estimate $\widehat{x}_{k \mid k}$ of Kalman filtering is the LMSE estimate. By Lemma 2, we have

$$
\widehat{x}_{k \mid k}=E x_{k}+\operatorname{Cov}\left(x_{k}, Y_{k}\right) \operatorname{Var} Y_{k}^{\dagger}\left(Y_{k}-E Y_{k}\right)
$$

where

$$
Y_{k}=\left(y_{1}^{T}, y_{2}^{T}, \ldots, y_{k}^{T}\right)
$$

Multiplying both sides of (11) by the same matrix $D_{k}$,

$$
\begin{aligned}
D_{k} \widehat{x}_{k \mid k}= & D_{k}\left[E x_{k}+\operatorname{Cov}\left(x_{k}, Y_{k}\right) \operatorname{Var} Y_{k}^{\dagger}\left(Y_{k}-E Y_{k}\right)\right] \\
= & D_{k} E x_{k}+D_{k} E\left(x_{k}-E x_{k}\right) \\
& \cdot\left(Y_{k}-E Y_{k}\right)^{T} \operatorname{Var} Y_{k}^{\dagger}\left(Y_{k}-E Y_{k}\right) \\
= & E D_{k} x_{k}+E\left(D_{k} x_{k}-D_{k} E x_{k}\right) \\
& \cdot\left(Y_{k}-E Y_{k}\right)^{T} \operatorname{Var} Y_{k}^{\dagger}\left(Y_{k}-E Y_{k}\right) \\
= & E D_{k} x_{k}+E\left(D_{k} x_{k}-E D_{k} x_{k}\right) \\
& \cdot\left(Y_{k}-E Y_{k}\right)^{T} \operatorname{Var} Y_{k}^{\dagger}\left(Y_{k}-E Y_{k}\right) \\
= & E d_{k}+E\left(d_{k}-E d_{k}\right)\left(Y_{k}-E Y_{k}\right)^{T} \operatorname{Var}_{k}^{\dagger}\left(Y_{k}-E Y_{k}\right) \\
= & d_{k}+E\left(d_{k}-d_{k}\right)\left(Y_{k}-E Y_{k}\right)^{T} \operatorname{Var} Y_{k}^{\dagger}\left(Y_{k}-E Y_{k}\right) \\
= & d_{k}+E 0\left(Y_{k}-E Y_{k}\right)^{T} \operatorname{Var}_{k}^{\dagger}\left(Y_{k}-E Y_{k}\right) \\
= & d_{k} .
\end{aligned}
$$

We complete the proof.

Remark 5. By Corollary 4, Gupta's conclusion and the conclusion in Theorem 3 are consistent. Since $\widehat{x}_{k \mid k}$ satisfies the constraint, the projection of $\widehat{x}_{k \mid k}$ onto the constraint set is still equal to itself. Thus, we show $\hat{x}_{k \mid k}=\tilde{x}_{k \mid k}$ in another way.

By Gupta's conclusion, although the pseudo measurement can make the estimate better than the estimate without the pseudo measurement in the case of mismatched process equation, we have to note that the model mismatch is also an important factor in estimation performance. In practice, it is quite possible for a large mismatch to result due to an extra constraint, especially when the constraint not only constrains the trajectory of target but also significantly changes its moving conditions. For example, if the unconstrained process equation originally presents a car as moving on a high way, then one discovers that it is moving on a countryside road. In this case, the velocity of the car and road noise would be very different; that is, the original process equation mismatches the present state significantly. In the next section, we will show through a numerical example that the pseudo measurement method is not always a good choice when the process equation mismatch is large.

\section{Numerical Examples}

In this section, simulations will be done for a dynamic system with equality constraints. We discuss the performances of the pseudo measurement method in both an exactly matched process equation and a mismatched process equation.

Example 1 (exactly matched process equation). A twodimension target tracking problem is considered. The target dynamic models are given as (1)-(2). The first two elements 
of the state vector are positions in two coordinates and the last two elements of the state vector are their velocities, respectively. The state transition matrices

$$
F_{k}=\left(\begin{array}{cccc}
1 & 0 & T & 0 \\
0 & 1 & 0 & 0 \\
0 & 0 & 1 & 0 \\
0 & 0 & 0 & 1
\end{array}\right)
$$

and the measurement matrix is given by

$$
H_{k}=\left(\begin{array}{llll}
1 & 0 & 0 & 0 \\
0 & 1 & 0 & 0
\end{array}\right) \text {. }
$$

The target starts at $\mathbf{x}_{0}=(50,10,1,1)^{T}, T=1$. The covariance matrices of the noises are given by

$$
R_{v_{k}}=\left(\begin{array}{cccc}
1 & 0 & 0 & 0 \\
0 & 0 & 0 & 0 \\
0 & 0 & 1 & 0 \\
0 & 0 & 0 & 1
\end{array}\right), \quad R_{\omega_{k}}=\left(\begin{array}{ll}
1 & 0 \\
0 & 1
\end{array}\right) .
$$

It is clear that the target moves along a line and the state satisfies the following equality constraint:

$$
D x_{k}=\left(\begin{array}{llll}
0 & 1 & 0 & 0
\end{array}\right) x_{k}=10 .
$$

Using 100 Monte-Carlo runs, we can evaluate the estimation performance of an algorithm by estimating the second moment of the tracking error:

$$
\begin{array}{r}
E_{k}^{2}=\frac{1}{100} \sum_{j=1}^{100}\left[\left(x_{k \mid k}^{(j)}(1)-x_{k}(1)\right)^{2}+\left(x_{k \mid k}^{(j)}(2)-x_{k}(2)\right)^{2}\right], \\
k=1,2, \ldots, 50 .
\end{array}
$$

Figure 1 compares the tracking errors of Kalman filtering with and without pseudo measurement. The performance of the pseudo measurement method is the same as that of Kalman filtering. The use of pseudo measurement for filtering is redundant.

Figure 2 shows the estimates of Kalman filtering and those of the pseudo measurement method are equal and satisfy the equality constraint.

Example 2 (mismatched process equation). The true dynamic equations of the states are the same as in Example 1. However, one case of a mistaken process equation would be

$$
x_{k+1}=\widetilde{F}_{k} x_{k}+\widetilde{v}_{k},
$$

where

$$
\widetilde{F}_{k}=\left(\begin{array}{cccc}
1 & 0 & T & 0 \\
0 & 1 & 0 & T \\
0 & 0 & 1 & 0 \\
0 & 0 & 0 & 1
\end{array}\right), \quad \widetilde{R}_{v_{k}}=\left(\begin{array}{cccc}
0.1 & 0 & 0 & 0 \\
0 & 0 & 0 & 0 \\
0 & 0 & 0.1 & 0 \\
0 & 0 & 0 & 0.1
\end{array}\right) .
$$

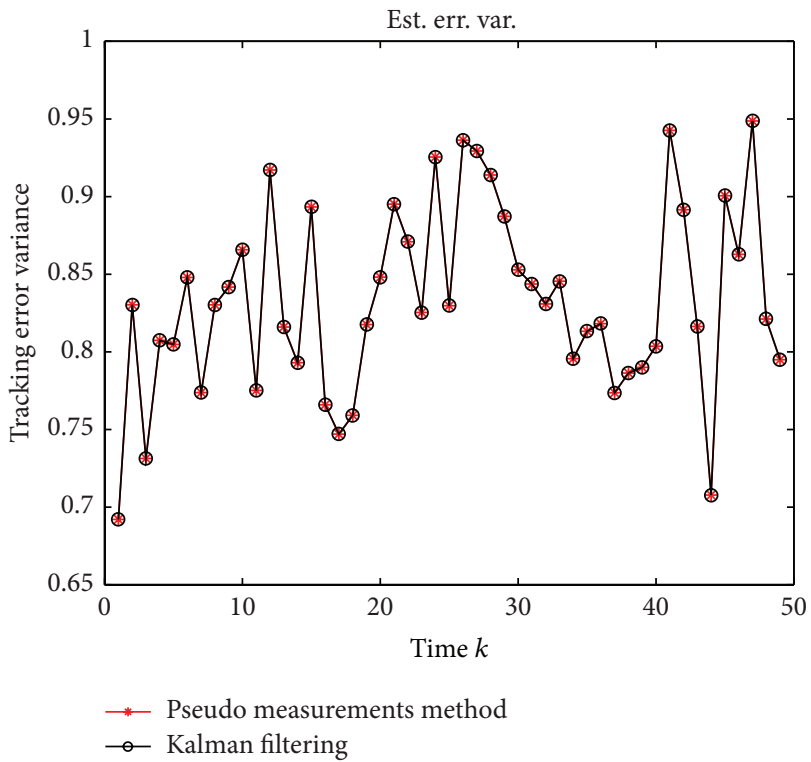

FIGURE 1: Comparison of Kalman filtering and the pseudo measurement method.

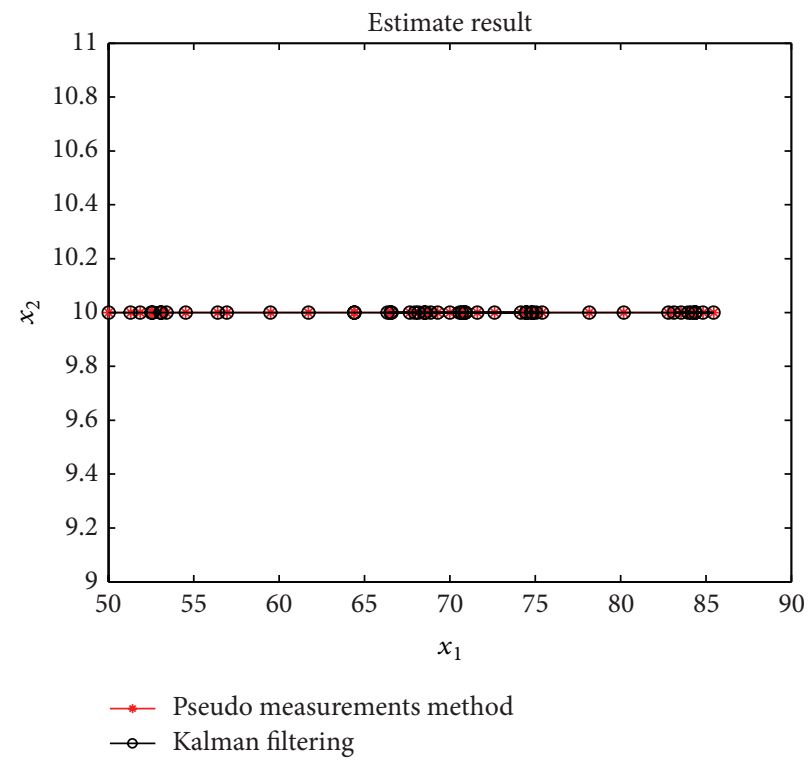

FIgURE 2: Estimates of Kalman filtering and the pseudo measurement method.

The target starts at $\mathbf{x}_{0}=(50,10,1,1)^{T}, T=1$. The measurement equation is the same as in Example 1. We get the additional information that the state satisfies the following equality constraint:

$$
D x_{k}=\left(\begin{array}{llll}
0 & 1 & 0 & 0
\end{array}\right) x_{k}=10 .
$$

In the following, we will compare the tracking errors of three algorithms, that is, standard Kalman filtering, the pseudo measurement method, and the measurement projection method. The measurement projection method directly projects the measurement onto the constraint set and takes this projection of the measurement as the estimate. 


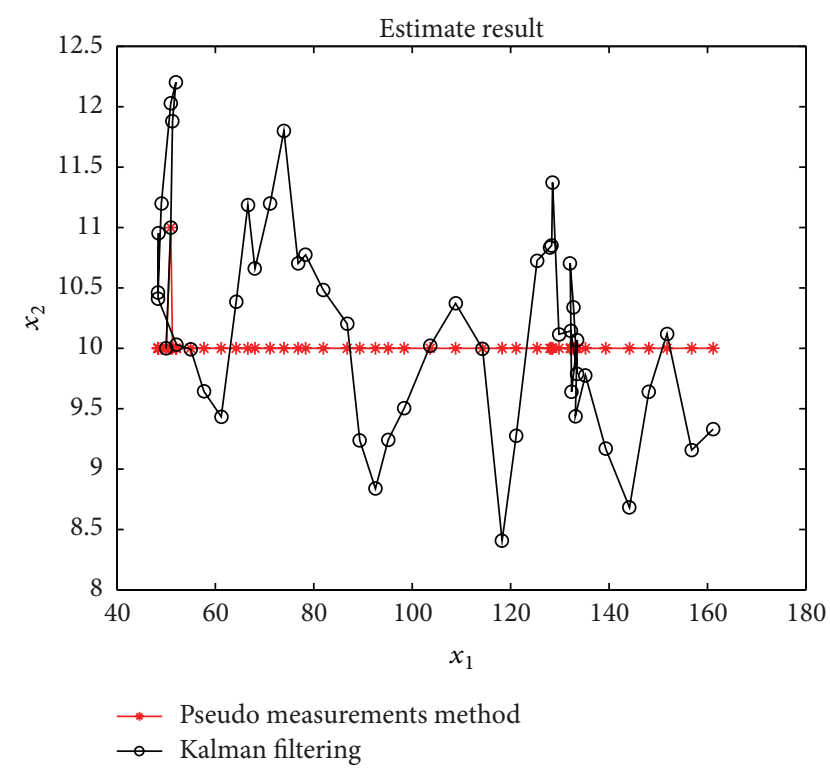

Figure 3: Estimates of Kalman filtering and the pseudo measurement method.

It is obvious that the measurement projection method is only a choice of state estimation and certainly not optimal in the MSE sense. However, the measurement projection method seems to be a better choice than the pseudo measurement method when the mismatch of the process equation is large.

Figure 3 shows that the estimates of the pseudo measurement method are really the projection of Kalman filtering onto the constraint set.

Using 100 Monte-Carlo runs, Figure 4 shows that the performance of the pseudo measurement method is better than that of Kalman filtering. However, the performances of both algorithms are worse than those of the measurement projection method. This is because the measurement projection method does not use the wrong process equation.

Remark 6. From this example, we should notice that the pseudo measurement method has a smaller error covariance than that of Kalman filtering, but the improvement is small and may not significantly neutralize the bad effects of the mismatched process equation. This is to say that we would rather discard the mismatched process equation than use it when the process equation mismatch is large.

\section{Conclusion}

In this paper, we discuss the pseudo measurement method in both an exactly matched process equation case and a mismatched process equation case. We show simply that the use of pseudo measurement for filtering is redundant when states in the process equation satisfy the equality constraint. When the process equation is mismatched, that is, its states do not satisfy the equality constraint, although the pseudo measurement method is better than the unconstrained state estimate, its performance is still not good enough to neutralize the

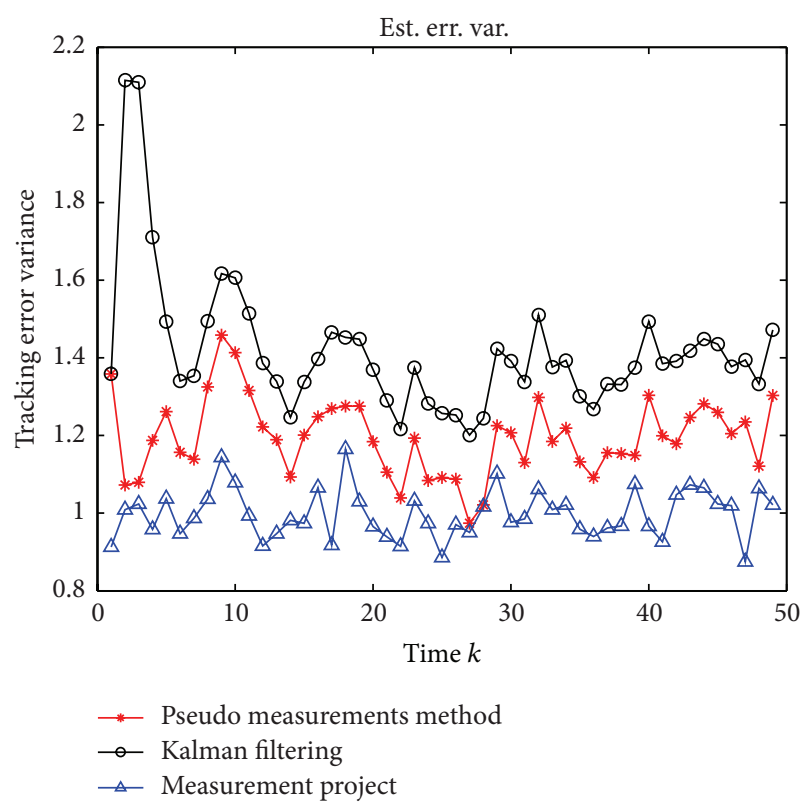

FIGURE 4: Comparison of the three methods.

bad effects of the mismatch process equation. This suggests that when the additional information not only constrains the trajectory of a target but also significantly changes its moving conditions, remodelling the process equation should be a better choice in the mismatched process equation case.

\section{Conflict of Interests}

The authors declare that there is no conflict of interests regarding the publication of this paper.

\section{Acknowledgment}

This research is supported by the NNSF of China (61201065 and 61273074).

\section{References}

[1] D. Massicotte, R. Z. Morawski, and A. Barwicz, "Incorporation of a positivity constraint into a Kalman-filter-based algorithm for correction of spectrometric data," IEEE Transactions on Instrumentation and Measurement, vol. 44, no. 1, pp. 2-7, 1995.

[2] A. T. Alouani and W. D. Blair, "Use of a kinematic constraint in tracking constant speed, maneuvering targets," IEEE Transactions on Automatic Control, vol. 38, no. 7, pp. 1107-1111, 1993.

[3] T. Kirubarajan, Y. Bar-Shalom, and K. R. Pattipati, "Ground target tracking with variable structure IMM estimator," IEEE Transactions on Aerospace and Electronic Systems, vol. 36, no. 1, pp. 26-46, 2000.

[4] M. Spong, S. Hutchinson, and M. Vidyasagar, Robot Modeling and Control, John Wiley \& Sons, 2005.

[5] W. Wen and H. F. Durrant-Whyte, "Model based active object localisation using multiple sensors," in Proceedings of the 
IEEE/RSJ International Workshop on Intelligent Robots and Systems (IROS '91), vol. 3, pp. 1448-1452, Osaka, Japan, November 1991.

[6] W. Wen and H. F. Durrant-Whyte, "Model-based multi-sensor data fusion," in Proceedings of the IEEE International Conference on Robotics and Automation, pp. 1720-1726, May 1992.

[7] J. Porrill, "Optimal combination and constraints for geometrical sensor data," International Journal of Robotics Research, vol. 7, no. 6, pp. 66-77, 1988.

[8] A. Pizzinga, "Constrained Kalman filtering: additional results," International Statistical Review, vol. 78, no. 2, pp. 189-208, 2010.

[9] T. L. Chia, P.-C. Chow, and H. J. Chizeck, "Recursive parameter identification of constrained systems: an application to electrically stimulated muscle," IEEE Transactions on Biomedical Engineering, vol. 38, no. 5, pp. 429-442, 1991.

[10] R. Zanetti, M. Majji, R. H. Bishop, and D. Mortari, "Normconstrained kalman filtering," Journal of Guidance, Control, and Dynamics, vol. 32, no. 5, pp. 1458-1465, 2009.

[11] R. J. Hewett, M. T. Heath, M. D. Butala, and F. Kamalabadi, "A robust null space method for linear equality constrained state estimation," IEEE Transactions on Signal Processing, vol. 58, no. 8, pp. 3961-3971, 2010.

[12] $\mathrm{H}$. Wu, W. Wang, and $\mathrm{H}$. Ye, "Model reduction based setmembership filtering with linear state equality constraints," IEEE Transactions on Aerospace and Electronic Systems, vol. 49, no. 2, pp. 1391-1399, 2013.

[13] T. L. Song, J. Y. Ahn, and C. Park, "Suboptimal filter design with pseudomeasurements for target tracking," IEEE Transactions on Aerospace and Electronic Systems, vol. 24, no. 1, pp. 28-39, 1988.

[14] M. Tahk and J. L. Speyer, "Target tracking problems subject to kinematic constraints," in Proceedings of the IEEE Conference on Decision and Control, pp. 1058-1059, 1988.

[15] M. Tahk and J. L. Speyer, "Target tracking problems subject to kinematic constraints," IEEE Transactions on Automatic Control, vol. 35, no. 3, pp. 324-326, 1990.

[16] H. E. Doran, "Constraining Kalman filter and smoothing estimates to satisfy time-varying restrictions," The Review of Economics and Statistics, vol. 74, no. 3, pp. 568-572, 1992.

[17] D. Simon, "Kalman filtering with state constraints: a survey of linear and nonlinear algorithms," IET Control Theory and Applications, vol. 4, no. 8, pp. 1303-1318, 2010.

[18] Z. S. Duan and X. R. Li, "The role of pseudo measurements in equality-constrained state estimation," IEEE Transactions on Aerospace and Electronic Systems, vol. 49, no. 3, pp. 1654-1666, 2013.

[19] K. Mahata and T. Söderström, "Improved estimation performance using known linear constraints," Automatica, vol. 40, no. 8, pp. 1307-1318, 2004.

[20] D. Simon and T. L. I. Chia, "Kalman filtering with state equality constraints," IEEE Transactions on Aerospace and Electronic Systems, vol. 38, no. 1, pp. 128-136, 2002.

[21] S. Ko and R. R. Bitmead, "State estimation for linear systems with state equality constraints," Automatica, vol. 43, no. 8, pp. 1363-1368, 2007.

[22] N. Gupta and R. Hauser, "Kalman filtering with equality and inequality state constraints," Oxford University Numerical Analysis Group Technical Report, University of Oxford, Oxford, UK, 2007.

[23] D. Simon and D. L. Simon, "Constrained Kalman filtering via density function truncation for turbofan engine health estimation," International Journal of Systems Science, vol. 41, no. 2, pp. 159-171, 2010.
[24] N. Gupta, "Kalman Filtering in the presence of state space equality constraints," in Proceedings of the IEEE 26th Chinese Control Conference (CCC '07), pp. 107-113, Hunan, China, July 2007.

[25] N. Gupta and R. Hauser, "Mathematically equivalent approaches for equality constrained kalman filtering," Oxford University Numerical Analysis Group Technical Report, Oxford University, Oxford, UK, 2009.

[26] B. O. S. Teixeira, J. Chandrasekar, L. A. B. Tôrres, L. A. Aguirre, and D. S. Bernstein, "State estimation for linear and non-linear equality-constrained systems," International Journal of Control, vol. 82, no. 5, pp. 918-936, 2009.

[27] B. D. O. Anderson and J. B. Moore, Optimal Filtering, PrenticeHall, Englewood Cliffs, NJ, USA, 1979. 


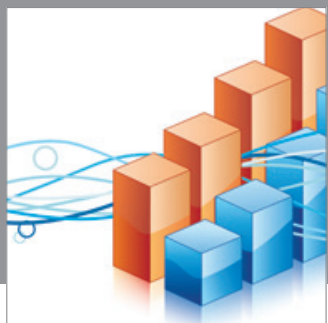

Advances in

Operations Research

mansans

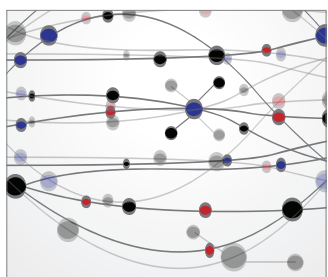

The Scientific World Journal
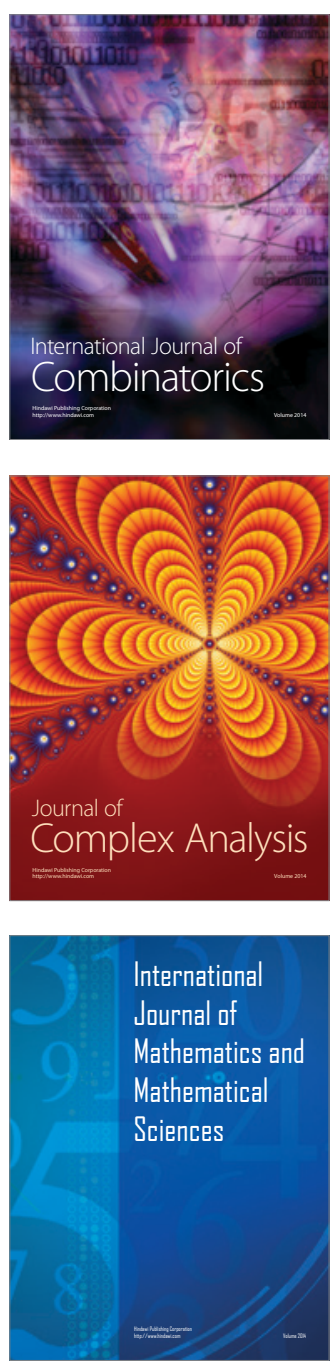
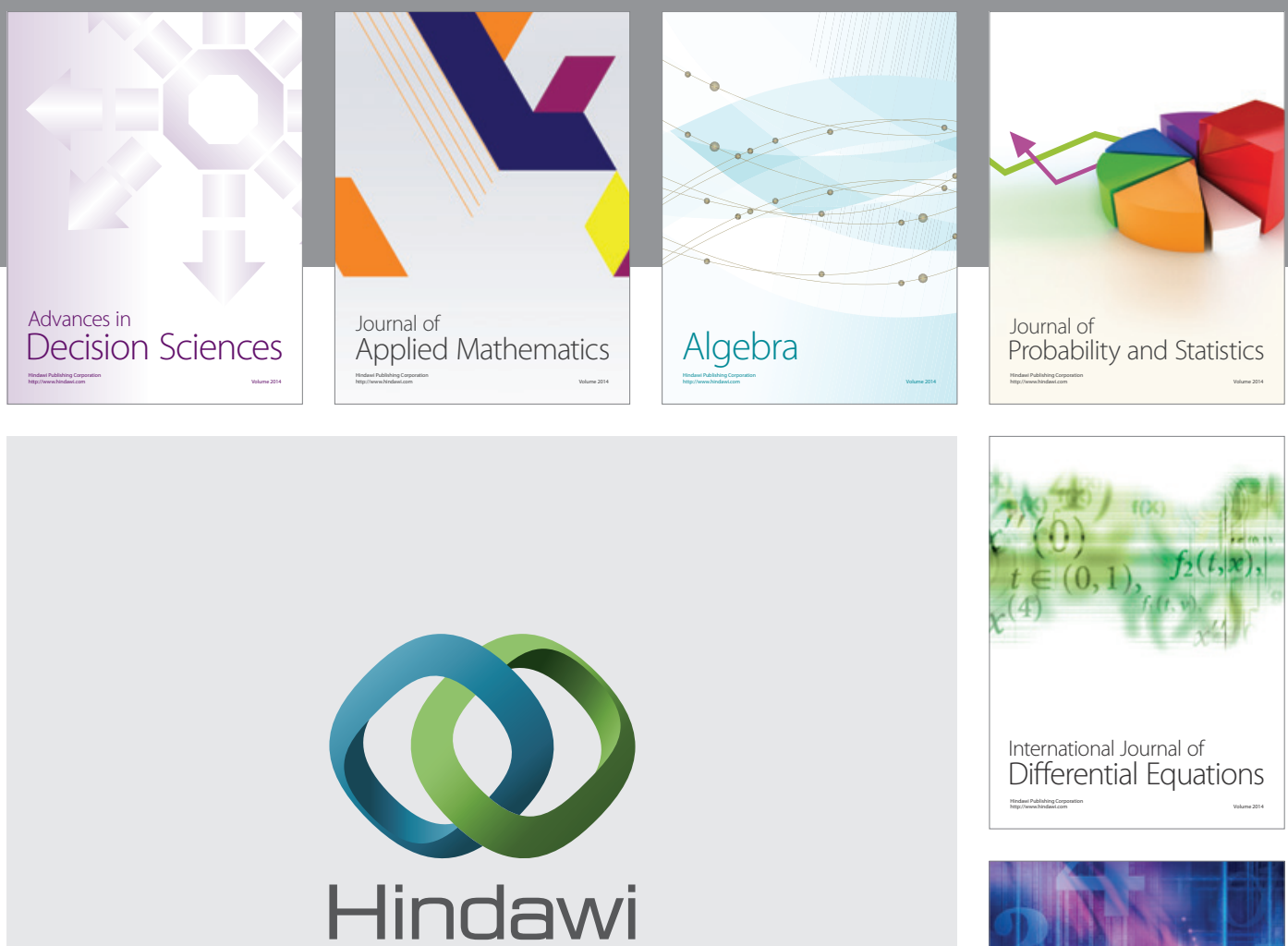

Submit your manuscripts at http://www.hindawi.com
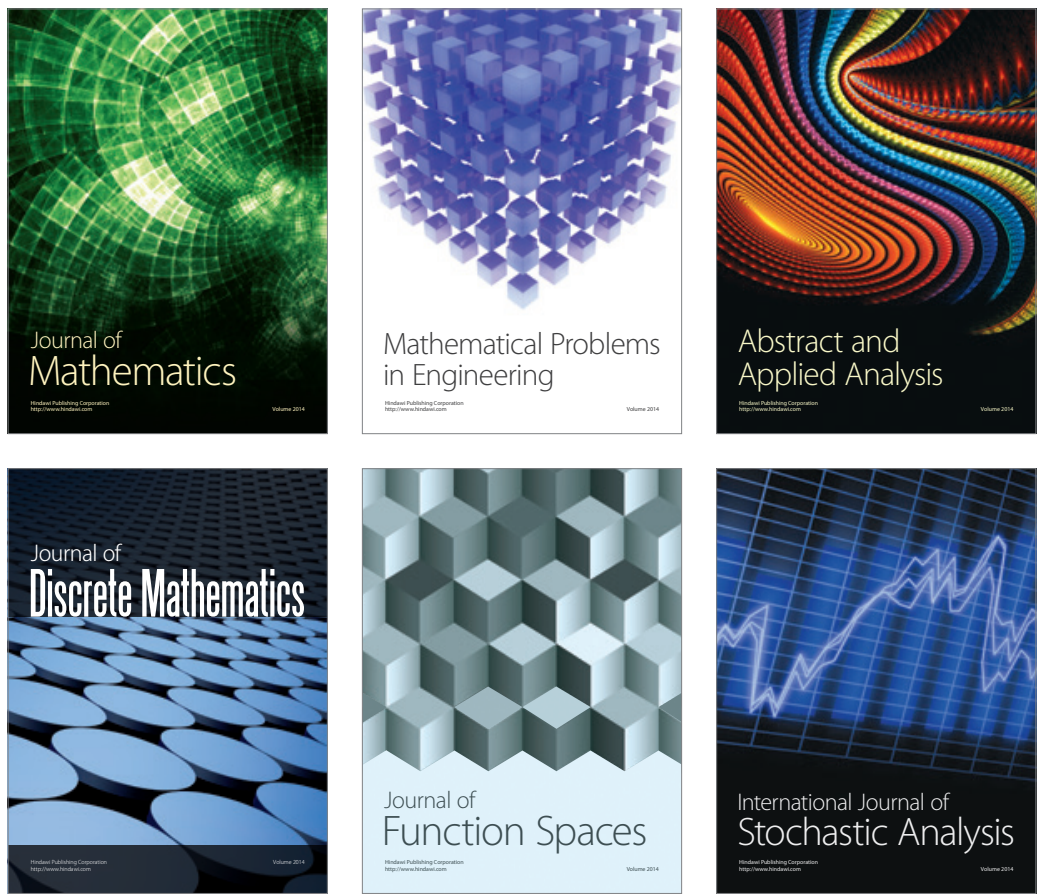

Journal of

Function Spaces

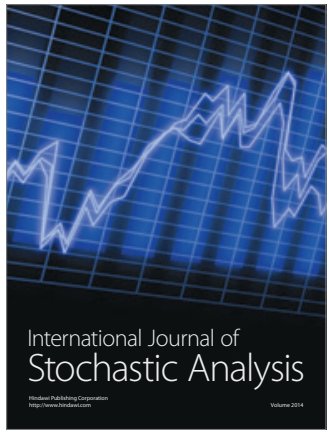

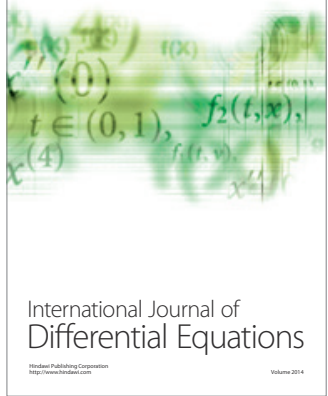
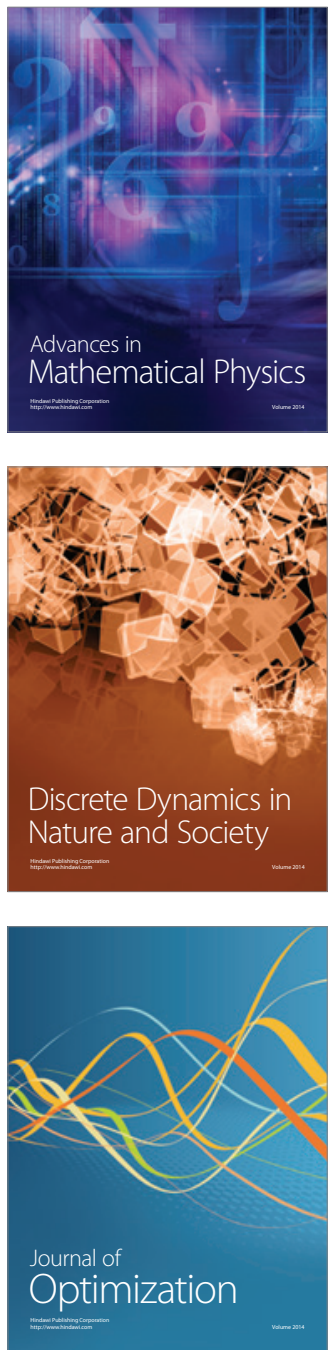